\title{
Validity of an Artificial Intelligence-Assisted Motion-Analysis System Using a Smartphone for Evaluating Weight-Bearing Activities in Individuals with Patellofemoral Pain Syndrome
}

\author{
Jun-Seok Kim, PT, MS; Yong-Wook Kim, PT, Ph.D; Young-Keun Woo, PT, Ph.D; Kyue-Nam Park, PT, Ph.D \\ Department of Physical Therapy, College of Medical Science, Jeonju University, Jeonju, South Korea
}

Background An artificial intelligence-assisted motion-analysis system without markers, OpenPose, is used to calculate joint angles in sports and medical analyses. Measuring the angles of the hip and knee joint in the frontal plane during the performance of weight-bearing activities is valuable in patients with patellofemoral pain syndrome (PFPS).

Purpose The purpose of this study was to assess the validity of OpenPose using a pre-trained human motion-tracking algorithm for measuring the angles of the hip and knee joint in the frontal plane during standing hip abduction, semi-squat movements, and forward step-down movements compared with marker-based three-dimensional motion analysis.

Study design Cross-sectional study

Methods Eight individuals with PFPS participated in the current study. To investigate the validity of OpenPose, the angles of the hip and knee in the frontal plane were measured simultaneously with a smartphone camera using the OpenPose library and Vicon as the gold-standard motionanalysis system while performing three weight-bearing activities. Pearson and Spearman correlation analysis was used to assess the validity of the OpenPose-based motion-analysis system.

Results Correlation coefficients ranged from 0.04 to 0.61 on the more symptomatic side and from 0.02 to 0.88 on the less symptomatic side for the three weight-bearing activities. When performing standing hip abduction and step-down movements, the validity of the measurements of hip abduction was fair or good. When performing semi-squat movements, the validity of the knee abduction measurements was fair.

Conclusions The OpenPose-based motion-analysis system can provide fair or good level of J Musculoskelet Sci Technol 2021; 5(1): 34-40 Published Online Jun 30, 2021 pISSN 2635-8573 eISSN 2635-8581

Article History Received 30 Apr 2021 Revised 10 May 2021 (1st) Accepted 11 May 2021

\section{CONTACT}

knpark@jj.ac.kr Kyue-Nam Park, Department of Physical Therapy, College of Medical Science, Jeonju University, Jeonju, South Korea

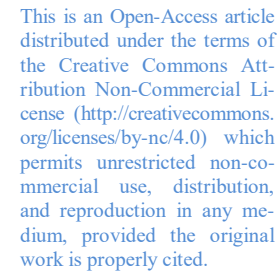
validity of measurements of frontal hip and knee angles during weight-bearing activities of individuals with PFPS in real environments and for remote rehabilitation.

Key words Artificial intelligence; Markerless motion analysis; Patellofemoral pain syndrome; Valgus; Validity.

\section{INTRODUCTION}

Patellofemoral pain syndrome (PFPS) is one of the most common forms of knee pain around the anterior aspect of the knee. ${ }^{1}$ Activities such as prolonged sitting, descending stairs, and squatting often exacerbate pain in patients with PFPS because these functional activities increase the compressive force on the patellofemoral joint. ${ }^{2}$ Especially, greater hip adduction or knee abduction in the frontal plane may increase the stress to the patellofemoral joint during walking and single leg standing, leading to PFPS. ${ }^{3}$ People with PFPS had greater angle of knee valgus during squat than asymptomatic people. ${ }^{4}$ In prospective study, people who developed PFPS had greater hip adduction during single leg squat than people who did not develop PFPS, suggesting that excessive hip adduction in the frontal plane may be considered as one 
of the causes of PFPS. ${ }^{5}$

A set of reflective markers and inertial sensors have been used for investigations via a kinematic analysis system. ${ }^{6}$ However, these markers are rarely used for three-dimensional (3D) motion analysis in clinics due to their significant financial, spatial, and temporal costs. ${ }^{7}$ Markerless motion-capture techniques can expand the applicability of human motion-capture systems and do not require special equipment for motion tracking or laborious processing, thus leading to a considerable decrease in preparation time in terms of recording and analyzing motion data. ${ }^{8,9}$ Microsoft Kinect and Intel RealSense systems with low-cost depth cameras are often used in markerless 3D motion analysis due to their high accuracy. ${ }^{10}$

New advances in markerless motion analysis have been achieved using cameras in mobile smartphones, tablets, and PCs; thus, an extra 3D depth camera is not required. ${ }^{11}$ An open-source system named OpenPose has been launched on TensorFlow, an open-source platform for machine learning. OpenPose can automatically identify anatomical points and segments of the human body using an artificial-intelligence engine that includes robust machine-learning algorithms and a pre-trained human motion-tracking algorithm. ${ }^{9}$ A previous study suggested that OpenPose can be used as a two-dimensional (2D) markerless motion-analysis system for musculoskeletal assessment that can calculate ranges of motion and human postures without manually marking key points on the body. ${ }^{9}$ If so, an OpenPose-based motionanalysis system will be valuable for evaluating the frontal range of hip and knee motion in individuals with PFPS, which is usually challenging.

Measurements of the frontal hip and knee angles and the functionality of the hip abductor during hip abduction, stepdown, and squat activities have been used to identify risk factors for PFPS. ${ }^{12,13}$ However, the accuracy of the OpenPose-based motion-analysis system has not yet been fully validated for individuals with PFPS undergoing knee rehabilitation. Therefore, the purpose of this study was to assess the validity of the OpenPose-based motion-analysis system compared with 3D motion analysis for measuring the frontal range of the hip and knee joints during weightbearing activities in individuals with PFPS.

\section{METHODS}

\section{Participants}

Eight subjects with PFPS participated in this study. The inclusion criteria were knee pain intensity of $>3$ points on the visual analog scale (0-10 points), Western Ontario and McMaster Universities Osteoarthritis Index (WOMAC) score of $>30$ points, presence of retropatellar or anterior knee pain, and pain exacerbation with at least two of the following activities: prolonged sitting, squatting, ascending or descending stairs, and kneeling. ${ }^{14-16}$ Participants were excluded if they had a history of surgery or musculoskeletal disorder within the previous year; had diseases that affect balance or walking (vestibular and neurological disorders), osteoarthritis, or rheumatoid arthritis; or could not walk independently without a walking aid. ${ }^{17}$ All subjects consented to participate in this study and provided informed consent. This study was approved by the Jeonju University Institutional Review Board (jjIRB-191115-HR-2019-1108).

\section{Instrumentation}

\section{1) Vicon (Marker based motion analysis)}

The Vicon 3D motion-analysis system (Vicon MX System; Oxford Metrics, Oxford, UK) was used to investigate the validity of the TensorFlow-based application. The sampling rate was $100 \mathrm{~Hz}$. Six high-speed cameras were set up in the laboratory and sixteen $16-\mathrm{mm}$ reflective markers were secured using double-sided tape to the skin or with tightfitting pants on the anterior superior iliac spine, posterior superior iliac spine, lateral thighs, lateral femoral epicondyles, lateral shanks, lateral malleoli, calcaneus, and the dorsal surface of the second metatarsal of the feet. After calibration, Vicon Nexus version 1.8.5 software was used to capture kinematic parameters while the participants performed standing hip abduction, semi-squat, and step-down movements (Figure 1). Raw kinematic data were filtered using fourth-order Butterworth filters with a cut-off frequency of $6 \mathrm{~Hz}$ and exported as a CSV file for statistical analysis.

\section{2) OpenPose (markerless motion analysis)}

OpenPose is a popular open-source 2D pose-estimation system that can identify joint centers with a web camera or smartphone camera using a convolutional neural network and supervised learning. The OpenPose system can recognize joint positions such as the nose, neck, eye, ear, shoulder, elbow, wrist, hip, knee, and ankle, and estimate joint kinematics automatically without markers. ${ }^{18}$ To measure the frontal angle of the hip and knee, markerless motion capture was performed using a smartphone camera (Samsung Galaxy S8, Samsung Electronics Co., Ltd., Suwon, South Korea) on the tripod without tilting, which was placed on a $20-\mathrm{cm}$ tripod at a height of $100 \mathrm{~cm}$ in the frontal plane of the subject. The frontal plane angles of the hip and knee joints were analyzed based on the OpenPose library in the Python environment. The sampling rate was $30 \mathrm{~Hz}$. The raw kine- 


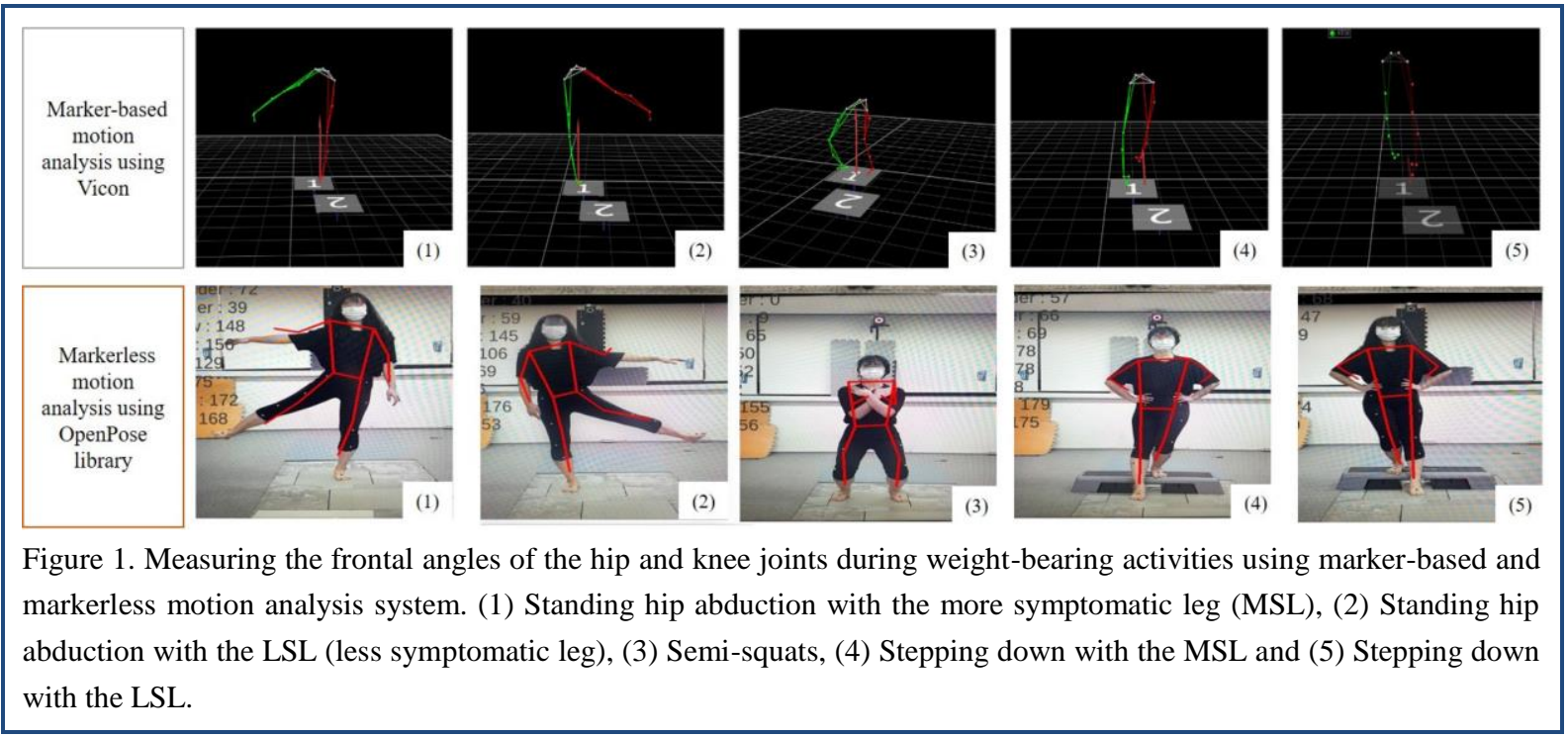

matic data were smoothed using a zero-lag fourth-order Butterworth low-pass filter. The cut-off frequency of the filter was $2 \mathrm{~Hz}$. The OpenPose system automatically calculates the relative angle between two vectors during hip abduction, semi-squat movements, and step-down movements. The feature points of the hip and knee joints were estimated using OpenPose during weight-bearing activities recorded using a smartphone camera (Figure 1).

\section{Procedure}

Before data collection using Vicon and OpenPose, the participants reported their height, weight, age, medical history, leg length, knee width, ankle width, PFPS intensity in both knees on a visual analog scale ( $0-10$ points), PFPS duration, and level of disability using the WOMAC (Table 1).

Participants randomly performed three weight-bearing activities at a self-selected speed in a standing position (hip abduction with both legs, semi-squats, and stepping down

Table 1. Subject characteristics

\begin{tabular}{cc}
\hline Characteristic & Value \\
\hline Gender $(\mathrm{M} / \mathrm{F})$ & $2 / 6$ \\
Age (years) & $25.3 \pm 4.1$ \\
\hline Height $(\mathrm{cm})$ & $167.0 \pm 9.9$ \\
Weight $(\mathrm{kg})$ & $63.9 \pm 20.3$ \\
WOMAC & $54.3 \pm 9.6$ \\
VAS $(\mathrm{cm})$ & $4.0 \pm 1.1$ \\
\hline
\end{tabular}

Data are expressed as mean \pm standard deviation.

Abbreviations: WOMAC, The Western Ontario and McMaster Universities Osteoarthritis Index; VAS, visual analogue scale. with both legs). Each participant practiced the weightbearing activities three times for familiarization and were allowed a rest period of 1 minute after each activity. Three trials of each activity were recorded simultaneously using both Vicon and OpenPose. The more symptomatic leg (MSL) and less symptomatic leg (LSL) were divided based on the VAS. For standing hip abduction with the MSL and LSL, the starting position was standing with both arms and legs straight while looking straight forward. The participant was asked to abduct the arm of the test side to $90^{\circ}$ to make the activity more unstable than hip abduction alone. The participant was then asked to open their leg as wide as they could. To perform semi-squats, the participant started in a standing position with their arms folded across the chest and the feet shoulder-width apart, looking straight ahead. The participant was then asked to flex both knees until an angle of $45^{\circ}$ was reached. To perform step-down movements with the MSL and LSL, the participant stood with their feet shoulder-width apart and their arms folded and positioned their toes toward the front end of the step, which was set at a height of $20 \mathrm{~cm}$ regardless of the length of their leg. A previous study showed no significant difference in the intensity of knee pain between three step heights $(8,14$, and $20 \mathrm{~cm}$ ) in patients with PFPS. ${ }^{19,20}$ The participant was asked to flex the knee of the tested side until the heel of the nontested limb touched the floor without putting weight on the heel and then return to the starting position.

To investigate the validity of the measurements, kinematic data for the hip and knee in the frontal plane were recorded simultaneously using Vicon and OpenPose during the three weight-bearing activities in the aforementioned laboratory setting (Figure 1). The peak angles of hip and knee joints in the frontal plane were calculated and the mean values of 
three trials of weight-bearing activities were used for the analyses.

\section{Statistical analysis}

The data were tested for normality using the ShapiroWilk normality test. ${ }^{21}$ Pearson and Spearman correlation analysis was used to assess the validity of the OpenPosebased motion-analysis system by comparing measurements of the hip and knee joint angles in the frontal plane between OpenPose and Vicon. The correlation coefficients were interpreted based on Swinscow's classification, as follows: $0.00-0.39$, very weak to weak correlation; $0.40-0.59$ fair to moderate correlation; $0.60-0.79$ good correlation; and 0.80 1.0 strong correlation. ${ }^{22}$ Statistical analyses were performed using SPSS software (ver. 26.0; IBM Corp, Armonk, NY, USA). The significance threshold was set at $p<0.05$.

\section{RESULTS}

Correlation coefficients ranged from 0.04 to 0.61 for the MSL and 0.02 to 0.88 for the LSL when participants were performing the three weight-bearing activities (Table 2).

\section{1) Standing hip abduction with the MSL}

When performing standing hip abduction with the MSL (unsupported side), the validity of the measurements of hip abduction and knee abduction for the MSL was fair and weak, respectively. For the LSL (supported side), the validity of the measurements of hip abduction and knee abduction was weak and good, respectively (Table 2).

\section{2) Standing hip abduction with the LSL}

When performing standing hip abduction with the LSL (unsupported side), the validity of the measurements of both hip abduction and knee abduction for the LSL was fair. For the MSL (supported side), the validity of the measurements of both hip abduction and knee abduction was weak (Table 2).

\section{3) Semi-squats}

When performing semi-squats, the validity of the measurements of hip abduction and knee abduction for the MSL was weak and fair, respectively. The validity of the measurements of both hip abduction and knee abduction for the LSL was fair (Table 2).

\section{4) Stepping down with the MSL}

When stepping down with the MSL (unsupported side), the validity of the measurements of hip abduction and knee abduction for the MSL was good and weak, respectively. The validity of the measurements of both hip abduction and knee abduction for the LSL (supported side) was weak and strong, respectively (Table 2).

\section{5) Stepping down with the LSL}

When stepping down with the LSL (unsupported side), the validity of the measurements of hip abduction and knee abduction for the LSL was weak and fair, respectively. The validity of the measurements of both hip abduction and knee abduction for the MSL (supported side) was fair (Table 2).

Table 2. The validity of OpenPose-based motion-analysis system for measuring the frontal angles of the hip and knee joints compared with three-dimensional motion analysis

$(n=8)$

\begin{tabular}{cccc}
\hline Weight-bearing activity & Range of motion & \multicolumn{2}{c}{ Correlation coefficient $(P$ value $)$} \\
\cline { 2 - 4 } & & MSL & LSL \\
\hline Standing hip abduction with the MSL & Hip abduction & $0.606(0.11)^{+}$ & $0.344(0.40)^{\#}$ \\
Standing hip abduction with the LSL & Knee abduction & $-0.314(0.45)^{\#}$ & $0.765(0.03)^{\#^{*}}$ \\
& Hip abduction & $0.390(0.34)^{+}$ & $0.500(0.21)^{\#}$ \\
Semi-squats & Knee abduction & $-0.036(0.93)^{\#}$ & $0.476(0.23)^{\#}$ \\
& Hip adduction & $0.253(0.55)^{+}$ & $0.550(0.16)^{+}$ \\
Stepping down with the MSL & Knee abduction & $0.400(0.33)^{+}$ & $0.318(0.44)^{+}$ \\
Stepping down with the LSL & Hip adduction & $0.632(0.09)^{+}$ & $-0.091(0.83)^{+}$ \\
& Knee abduction & $0.287(0.49)^{\#}$ & $-0.882(0.01)^{\# *}$ \\
\hline
\end{tabular}

${ }^{+}$Pearson correlation coefficient; ${ }^{\#}$ Spearman correlation coeeficient; $* P<0.05$.

Abbreviations: MSL, more symptomatic leg; LSL, less symptomatic leg. 


\section{DISCUSSION}

The purpose of the current study was to investigate the validity of measurements provided by an OpenPose-based motion-analysis system for the angles of the hip and knee joint in the frontal plane during standing hip abduction, semi-squat, and step-down movements in individuals with PFPS. We found that the validity of the measurements ranged from weak to strong. Although markerless motionanalysis systems are less accurate than marker-based motion-analysis systems, the former system is more suitable for use in clinical rehabilitation and sports, where it is difficult to perform marker-based motion analysis. ${ }^{23}$

A previous study reported that markerless motion analysis provided measurements with low validity, as tracking using an automated skeleton and relatively low camera sample rates was difficult. ${ }^{24}$ In addition, a recent systematic review reported that the outcomes of low-cost video-based motion-analysis and 3D motion-analysis systems exhibited "poor" ( $\mathrm{r}=0.025)$ to "strong" agreement $(\mathrm{r}=0.992) .{ }^{25}$ Current results also showed measurement validity ranging widely from weak $(\mathrm{r}=0.02)$ to strong $(\mathrm{r}=0.88)$. OpenPose was shown to provide measurements of sagittal angles and frontal angles of the hip and knee in a gait analysis of healthy participants that exhibited good and poor agreement, respectively, with measurements provided by $3 \mathrm{D}$ motionanalysis systems. ${ }^{26}$ The low accuracy of the current results, which is consistent with that in a previous study, may be because we measured frontal angles of the hip and knee when the participants were performing weight-bearing and functional activities. In addition, our participants comprised a patient group with PFPS. Although we did not compare hip and knee angles between people with and without PFPS, we confirmed that measurements of the LSL were more accurate than those of the MSL. The genu valgum angle was larger for the MSL than for the LSL. With a larger genu valgum angle, the sides of the knees are closer together, making it difficult to detect key points of the hip and knee using video-based OpenPose programming. By using 3D depth cameras built into smartphones in the future, we should be able to address this technical limitation and produce more valid measurements for PFPS patients that will complement the measurement of frontal hip and knee angles performed by clinicians. However, further studies are required to verify if this is possible.

We obtained measurements of the angle of hip abduction with good and fair validity for the MSL and LSL, respectively, during standing hip abduction. OpenPose measurements of the frontal plane of hip abduction/adduction in walking healthy participants were previously found to be poorly correlated with Vicon measurements, although measurements of the hip angle in the sagittal plane were strongly correlated. ${ }^{27}$ OpenPose captures motion data using only one digital camera, so angles in a frontal or sagittal plane with a transverse plane rotation were not measured with high accuracy in a previous study. ${ }^{27}$ In the current study, although we asked participants to perform hip abduction, it is difficult for participants with PFPS to perform only hip abduction without hip rotation or flexion/extension. These complex motions in multiple planes during standing hip abduction might have decreased the accuracy of the measurements compared to those from Vicon.

When performing semi squat and step down, weak to good validity for the angle of hip adduction and knee abduction of MSL and LSL sides were showed in current study. PFPS patients demonstrated excessive adduction and internal rotation of hip during weight-bearing activities such as single leg squat and step down. ${ }^{13,28}$ We found the occurrence of hip internal rotation when performing semi squat and step down based on the data obtained by Vicon. Motion in transverse plane may lead to lower accuracy in OpenPose motion analysis, compared to 3D motion analysis system during semi squat and step down. When performing semi squat, more trunk forward bending with hip flexion was occurred than step down and standing hip abduction, which make difficulty to detect the hip joint as the key points when using OpenPose library, leading to low validity. Interestingly, when performing step down, fair and strong validity were showed for angle of knee abduction in each supported side. Clinicians used to measure the angle of genu valgus in order to confirm the risk factors of knee injury and give feedback when applying valgus control intervention in weight bearing position. ${ }^{29}$ Thus, it is meaningful that OpenPose system with fair and strong validity of knee abduction during step down can be used for patients with PFPS.

Our study had some limitations. The participants were relatively young. Therefore, our findings may not be applicable to older populations. Additionally, only eight participants with PFPS were analyzed to assess the validity of the OpenPose-based motion-analysis system. Thus, we suggest using markerless motion analysis with caution when highly accurate assessments of kinematic variables are necessary until further studies with a larger sample size are available to assess the validity of such systems. Although the validity of most measurements was not strong in the current study, the OpenPose motion-analysis system has been demonstrated to have almost perfect reliability. ${ }^{30}$ If the OpenPose motion-analysis system can sufficiently discriminate between people with and without PFPS or reveal pre- and 
post-intervention clinical differences despite producing measurements with weak to strong validity, the markerless system can be clinically useful.

\section{CONCLUSIONS}

Our results support the use of the OpenPose-based motion-analysis system as a physical function assessment tool to investigate the frontal hip and knee angles of patients with PFPS due to its cost- and time-effectiveness, ease of use outside the laboratory, and utility for remote rehabilitation. However, to enhance the utility of the OpenPose-based motion-analysis system, the errors between the true values and data obtained using OpenPose programming should be reduced. Based on our findings, future studies could assess whether the OpenPose-based motion-analysis system can be used to discriminate between patients with and without PFPS while they are performing functional activities or monitor the outcomes and disease progression of PFPS.

\section{Key Points}

Question Can an artificial intelligence-assisted motion-analysis system using the OpenPose library provide valid measurements of individuals with patellofemoral pain syndrome (PFPS)?

Findings The OpenPose-based motion-analysis system provided fair or good level of validity of the frontal hip angle during the performance of standing hip abduction and stepdown movements. When semi-squat movements were performed, fairly valid measurements of the frontal knee angle were provided.

Meaning The OpenPose-based motion-analysis system is an easy-to-use assessment tool providing fair or good level validity of the frontal hip or knee angles of patients with PFPS in a real environment.

\section{Article information}

Conflict of Interest Disclosures: None.

Funding/Support: None.

Acknowledgment: None.

Ethic Approval: This study was approved by the Jeonju University Institutional Review Board (jjIRB-191115HR-2019-1108).

\section{REFERENCES}

1. Smith BE, Selfe J, Thacker D, et al. Incidence and prevalence of patellofemoral pain: a systematic review and meta-analysis. PLoS One. 2018;13(1):e0190892.
2. Rothermich MA, Glaviano NR, Li J, Hart JM. Patellofemoral pain: epidemiology, pathophysiology, and treatment options. Clin Sports Med. 2015;34(2):313-327.

3. Scholtes SA, Salsich GB. A dynamic valgus index that combines hip and knee angles: assessment of utility in females with patellofemoral pain. Int J Sports Phys Ther. 2017;12(3):333-340.

4. Levinger P, Gilleard W, Coleman C. Femoral medial deviation angle during a one-leg squat test in individuals with patellofemoral pain syndrome. Phys Ther Sport. 2007;8(4):163-168.

5. Noehren B, Hamill J, Davis I. Prospective evidence for a hip etiology in patellofemoral pain. Med Sci Sports Exerc. 2013;45(6):1120-1124.

6. Cronin NJ, Rantalainen T, Ahtiainen JP, Hynynen E, Waller B. Markerless 2d kinematic analysis of underwater running: a deep learning approach. J Biomech. 2019;87:75-82.

7. Maykut JN, Taylor-Haas JA, Paterno MV, DiCesare CA, Ford KR. Concurrent validity and reliability of $2 \mathrm{~d}$ kinematic analysis of frontal plane motion during running. Int J Sports Phys Ther. 2015;10(2):136-146.

8. Mündermann L, Corazza S, Andriacchi TP. The evolution of methods for the capture of human movement leading to markerless motion capture for biomechanical applications. J Neuroeng Rehabil. 2006;3:6.

9. Colyer SL, Evans M, Cosker DP, Salo AIT. A review of the evolution of vision-based motion analysis and the integration of advanced computer vision methods towards developing a markerless system. Sports Med Open. 2018;4(1):24.

10. Carfagni M, Furferi R, Governi L, et al. Metrological and critical characterization of the intel d415 stereo depth camera. Sensors (Basel). 2019;19(3).

11. Jones A, Sealey R, Crowe M, Gordon S. Concurrent validity and reliability of the simple goniometer iphone app compared with the universal goniometer. Physiother Theory Pract. 2014;30(7):512-516.

12. Stickler L, Finley M, Gulgin H. Relationship between hip and core strength and frontal plane alignment during a single leg squat. Phys Ther Sport. 2015;16(1):66-71.

13. Meira EP, Brumitt J. Influence of the hip on patients with patellofemoral pain syndrome: a systematic review. Sports Health. 2011;3(5):455-465.

14. Hott A, Liavaag S, Juel NG, Brox JI. Study protocol: a randomised controlled trial comparing the long term effects of isolated hip strengthening, quadriceps-based training and free physical activity for patellofemoral pain syndrome (anterior knee pain). BMC Musculoskelet Disord. 2015;16:40. 
15. Nouri F, Raeissadat SA, Eliaspour D, Rayegani SM, Rahimi MS, Movahedi B. Efficacy of high-power laser in alleviating pain and improving function of patients with patellofemoral pain syndrome: a single-blind randomized controlled trial. J Lasers Med Sci. 2019;10(1): 37-43.

16. Nijs J, Van Geel C, Van der auwera C, Van de Velde B. Diagnostic value of five clinical tests in patellofemoral pain syndrome. Man Ther. 2006;11(1):69-77.

17. Cole MH, van den Hoorn W, Kavanagh JK, et al. Concurrent validity of accelerations measured using a tri-axial inertial measurement unit while walking on firm, compliant and uneven surfaces. PLoS One. 2014; 9(5):e98395.

18. Zago M, Luzzago M, Marangoni T, De Cecco M, Tarabini M, Galli M. 3d tracking of human motion using visual skeletonization and stereoscopic vision. Front Bioeng Biotechnol. 2020;8:181.

19. Lim EH, Kim ME, Kim SH, Park KN. Effects of posterior $\mathrm{x}$ taping on movement quality and knee pain intensity during forward-step-down in patients with patellofemoral pain syndrome. J Sports Sci Med. 2020;19(1): 224-230.

20. McClinton S, Donatell G, Weir J, Heiderscheit B. Influence of step height on quadriceps onset timing and activation during stair ascent in individuals with patellofemoral pain syndrome. J Orthop Sports Phys Ther. 2007;37(5):239-244.

21. Kim TH, Je WM, Hwang BH. Effects of pelvic compression belt on three-dimensional motions of pelvic and trunk during one leg standing. J Musculoskelet Sci Technol. 2019;3(1):22-25.

22. Swinscow TDV, Campbell MJ. Statistics at square one.
Bmj London; 2002.

23. Nakano N, Sakura T, Ueda K, et al. Evaluation of 3d markerless motion capture accuracy using openpose with multiple video cameras. Front Sports Act Living. 2020;2:50.

24. Clark RA, Mentiplay BF, Hough E, Pua YH. Threedimensional cameras and skeleton pose tracking for physical function assessment: a review of uses, validity, current developments and kinect alternatives. Gait Posture. 2019;68:193-200.

25. Parks MT, Wang Z, Siu KC. Current low-cost videobased motion analysis options for clinical rehabilitation: a systematic review. Phys Ther. 2019;99(10):1405-1425.

26. Stenum J, Rossi C, Roemmich RT. Two-dimensional video-based analysis of human gait using pose estimation. PLoS Comput Biol. 2021;17(4):e1008935.

27. Ota M, Tateuchi H, Hashiguchi T, Ichihashi N. Verification of validity of gait analysis systems during treadmill walking and running using human pose tracking algorithm. Gait Posture. 2021;85:290-297.

28. Powers CM. The influence of abnormal hip mechanics on knee injury: a biomechanical perspective. J Orthop Sports Phys Ther. 2010;40(2):42-51.

29. Emamvirdi M, Letafatkar A, Khaleghi Tazji M. The effect of valgus control instruction exercises on pain, strength, and functionality in active females with patellofemoral pain syndrome. Sports Health. 2019;11(3): 223-237.

30. Ota M, Tateuchi H, Hashiguchi T, et al. Verification of reliability and validity of motion analysis systems during bilateral squat using human pose tracking algorithm. Gait Posture. 2020;80:62-67. 